\title{
Systems biology analysis of mitogen activated protein kinase inhibitor resistance in malignant melanoma
}

\author{
Helma Zecena', Daniel Tveit ${ }^{1}$, Zi Wang ${ }^{2,3}$, Ahmed Farhat ${ }^{2}$, Parvita Panchal ${ }^{2}$, Jing Liư ${ }^{2,3}$, Simar J. Singh', \\ Amandeep Sanghera', Ajay Bainiwal', Shuan Y. Teo ${ }^{1}$, Frank L. Meyskens $\mathrm{Jr}^{2}$, Feng Liu-Smith ${ }^{2,4^{*}}$ \\ and Fabian V. Filipp ${ }^{1 *}$ (D)
}

\begin{abstract}
Background: Kinase inhibition in the mitogen activated protein kinase (MAPK) pathway is a standard therapy for cancer patients with activating BRAF mutations. However, the anti-tumorigenic effect and clinical benefit are only transient, and tumors are prone to treatment resistance and relapse. To elucidate mechanistic insights into drug resistance, we have established an in vitro cellular model of MAPK inhibitor resistance in malignant melanoma.

Methods: The cellular model evolved in response to clinical dosage of the BRAF inhibitor, vemurafenib, PLX4032. We conducted transcriptomic expression profiling using RNA-Seq and RT-qPCR arrays. Pathways of melanogenesis, MAPK signaling, cell cycle, and metabolism were significantly enriched among the set of differentially expressed genes of vemurafenib-resistant cells vs control. The underlying mechanism of treatment resistance and pathway rewiring was uncovered to be based on non-genomic adaptation and validated in two distinct melanoma models, SK-MEL-28 and A375. Both cell lines have activating BRAF mutations and display metastatic potential.

Results: Downregulation of dual specific phosphatases, tumor suppressors, and negative MAPK regulators reengages mitogenic signaling. Upregulation of growth factors, cytokines, and cognate receptors triggers signaling pathways circumventing BRAF blockage. Further, changes in amino acid and one-carbon metabolism support cellular proliferation despite MAPK inhibitor treatment. In addition, treatment-resistant cells upregulate pigmentation and melanogenesis, pathways which partially overlap with MAPK signaling. Upstream regulator analysis discovered significant perturbation in oncogenic forkhead box and hypoxia inducible factor family transcription factors.

Conclusions: The established cellular models offer mechanistic insight into cellular changes and therapeutic targets under inhibitor resistance in malignant melanoma. At a systems biology level, the MAPK pathway undergoes major rewiring while acquiring inhibitor resistance. The outcome of this transcriptional plasticity is selection for a set of transcriptional master regulators, which circumvent upstream targeted kinases and provide alternative routes of mitogenic activation. A fine-woven network of redundant signals maintains similar effector genes allowing for tumor cell survival and malignant progression in therapy-resistant cancer.
\end{abstract}

Keywords: Cancer systems biology, Precision medicine, Omics, RNA-Seq, Transcriptomics, Upstream regulator analysis, Transcription factor, Master regulator, Regulome, Non-genomic, Rewiring, Adaptation, Genetic selection, Drug resistance, Therapy resistance, Melanoma, Melanogenesis

\footnotetext{
* Correspondence: liufe@uci.edu; filipp@ucmerced.edu

${ }^{2}$ Department of Medicine, School of Medicine, Chao Family Comprehensive

Cancer Center, University of California Irvine, Irvine, CA 92697, USA

'Systems Biology and Cancer Metabolism, Program for Quantitative Systems

Biology, University of California Merced, 2500 North Lake Road, Merced, CA

95343, USA

Full list of author information is available at the end of the article
}

(c) The Author(s). 2018 Open Access This article is distributed under the terms of the Creative Commons Attribution 4.0 International License (http://creativecommons.org/licenses/by/4.0/), which permits unrestricted use, distribution, and reproduction in any medium, provided you give appropriate credit to the original author(s) and the source, provide a link to the Creative Commons license, and indicate if changes were made. The Creative Commons Public Domain Dedication waiver (http://creativecommons.org/publicdomain/zero/1.0/) applies to the data made available in this article, unless otherwise stated. 


\section{Background}

\section{Therapy resistance in cancer}

Cancer drug resistance is a major obstacle in achieving durable clinical responses with targeted therapies. This highlights a need to elucidate the underlying mechanisms responsible for resistance and identify strategies to overcome this challenge. In malignant melanoma, activating point-mutations in the mitogen activated protein kinase (MAPK) pathway in BRAF kinase (B-Raf protooncogene, serine/threonine kinase, Gene ID: 673) [1-3] made it possible to develop potent kinase inhibitors matched to genotyped kinase mutations in precision medicine approaches [4-6]. In tumors expressing the oncoprotein BRAF(V600E), the inhibitor molecules vemurafenib, dabrafenib, and encorafenib are designed to lock the ATP binding site into an inactive conformation of the kinase [4], the preferred state of wild-type RAF proteins. Trametinib and cobimetinib target MAP2K7 (MEK, mitogen-activated protein kinase kinase 7, Gene ID: 5609), the BRAF target and downstream effector molecule. In MAPK signaling, combinations of specific inhibitors have proven to be superior to singleagent regimens: BRAF inhibitors (BRAFi) in combination with MEK inhibitors (MEKi) improved survival compared to single MAPK inhibitors (MAPKi) [7-10]. However, many patients responding to small molecule inhibition of the MAPK pathway will develop resistance. Ultimately, disease progression will take place and patients relapse with lethal drug-resistant disease.

\section{Mechanism of resistance beyond mutations}

Acquired resistance has been shown to involve a diverse spectrum of oncogenic mutations in the MAPK pathway [11-15]. In addition, non-genomic activation of parallel signaling pathways has been noted [16]. Cell-to-cell variability in BRAF(V600E) melanomas generates drugtolerant subpopulations. Selection of genetically distinct, fully drug-resistant clones arise within a set of heterogeneous tumor cells surviving the initial phases of therapy due to drug adaptation [17]. Non-genomic drug adaptation can be accomplished reproducibly in cultured cells, and combination therapies that block adaptive mechanisms in vitro have shown promise in improving rates and durability of response [18]. Thus, better understanding of mechanisms involved in drug adaptation is likely to improve the effectiveness of melanoma therapy by delaying or controlling acquired resistance.

\section{Methods}

\section{Cellular models of malignant melanoma}

SK-MEL-28 and A375 are human skin malignant melanoma cell lines with BRAF(V600E) activation that are tumorigenic in xenografts [19-22] (HTB-72 and CRL1619, American Type Culture Collection, Manassas,
VA). The cell lines are maintained in DMEM medium supplemented with $10 \%$ fetal bovine serum and antibiotics (10-017-CV, 35-010-CV, 30-002-CI Corning, Corning, NY). All experimental protocols were approved by the Institutional Review Boards at the University of California Merced and Irvine. The study was carried out as part of IRB UCM13-0025 of the University of California Merced and as part of dbGap ID 5094 on somatic mutations in cancer and conducted in accordance with the Helsinki Declaration of 1975.

BRAFi-resistant (BRAFi-R) models were obtained by challenging cancer cell lines with incrementally increasing vemurafenib (PLX4032, PubChem CID: 42611257, Selleckchem, Houston, TX) concentrations in the culture media. Starting at $0.25 \mu \mathrm{M}$, which matched the naïve half maximal inhibitory concentration (IC50) of the parental cell lines, the vemurafenib concentrations were increased every 7 days in an exponential series up to 100-fold the naïve IC50 concentrations. Following this 6-week selection protocol, vemurafenib-adapted, cancer therapy resistance models were maintained in media supplemented with $5.0 \mu \mathrm{M}$ vemurafenib.

\section{Transcriptomic profiling and differential gene expression analysis}

Total RNA from malignant melanoma cells was extracted using a mammalian RNA mini preparation kit (RTN10-1KT, GenElute, Sigma EMD Millipore, Darmstadt, Germany) and then digested with deoxyribonuclease I (AMPD1-1KT, Sigma EMD Millipore, Darmstadt, Germany). Complementary DNA (cDNA) was synthesized using random hexamers (cDNA SuperMix, 95,048-500, Quanta Biosciences, Beverly, MA). The purified DNA library was sequenced using a HighSeq2500 (Illumina, San Diego, CA) at the University of California Irvine Genomics High-Throughput Facility. Purity and integrity of the nucleic acid samples were quantified using a Bioanalyzer (2100 Bioanalyzer, Agilent, Santa Clara, CA). Libraries for next generation mRNA transcriptome sequencing (RNA-Seq) analysis were generated using the TruSeq kit (Truseq RNA Library Prep Kit v2, RS-122-2001, Illumina, San Diego, $\mathrm{CA})$. In brief, the workflow involves purifying the poly-A containing mRNA molecules using oligo-dT attached magnetic beads. Following purification, the mRNA is chemically fragmented into small pieces using divalent cations under elevated temperature. The cleaved RNA fragments are copied into first strand cDNA using reverse transcriptase and random primers. Second strand cDNA synthesis follows, using DNA polymerase I and RNase $H$. The cDNA fragments are end repaired by adenylation of the $3^{\prime}$ ends and ligated to barcoded adapters. The products are then purified and enriched by nine cycles of PCR to create the final cDNA library subjected to 
sequencing. The resulting libraries were validated by qPCR and size-quantified by a DNA high sensitivity chip (Bioanalyzer, 5067-4626, Agilent, Santa Clara, CA). Sequencing was performed using 50 base pair read length, single-end reads, and more than $10^{7}$ reads per sample. Raw sequence reads in the file format for sequences with quality scores (FASTQ) were mapped to human reference Genome Reference Consortium GRCh38 using Bowtie alignment with an extended Burrows-Wheeler indexing for an ultrafast memory efficient alignment within the Tuxedo suite followed by Tophat to account for splice-isoforms [23, 24]. Read counts were scaled via the median of the geometric means of fragment counts across all libraries. Transcript abundance was quantified using normalized single-end RNA-Seq reads in read counts as well as reads per kilobase million (RPKM). Since single-end reads were acquired in the sequencing protocol, quantification of reads or fragments yielded similar results. Statistical testing for differential expression was based on read counts and performed using EdgeR in the Bioconductor toolbox [25]. Differentially expressed genes were further analyzed using Ingenuity Pathways Analysis (IPA, Qiagen, Rewood City, CA), classification of transcription factors (TFClass), and gene set enrichment analysis (GSEA, Broad Institute, Cambridge, MA) [26, 27]. For real-time quantitative polymerase chain reaction (RT-qPCR) validation of RNA-Seq signals of differentially expressed target genes in BRAFi-R melanoma cells, gene expression profiles were analyzed using the $\Delta \Delta$ threshold cycle (CT) method. Oligonucleotides spanning exon-exon-junctions of transcripts were used for RT-qPCR validation (Additional file 1: Table 1). Triple replicate samples were subjected to SYBR green (SYBR green master mix, PerfeCTa ${ }^{\circ} \mathrm{SYBR}^{\circ}$ Green FastMix, 95072-05k, Quanta Biosciences, Beverly, MA) RT-qPCR analysis in an Eco system (Illumina, San Diego). CT values were normalized using multiple housekeeping genes like actin beta (ACTB, Gene ID: 60), cyclophilin A (PPIA, peptidylprolyl isomerase A, Gene ID: 5478) and RNA polymerase II subunit A (POLR2A, GeneID: 5430).

\section{Inhibitor cytotoxicity studies}

Chemical BRAFi against BRAF(V600E), vemurafenib, was dissolved in dimethyl sulfoxide (DMSO, Sigma) as a $10.0 \mathrm{mM}$ stock solution and used in treatments in final concentrations between $0.01 \mu \mathrm{M}$ and $50.0 \mu \mathrm{M}$. Melanoma control experiments were carried out in the presence of equivalent amounts of DMSO solvent without drug. Cell viability was determined using a 2,3-bis(2-methoxy-4-nitro-5-sulfophenyl)-2H-tetrazolium-5-carboxanilide (XTT, X4626, Sigma EMD Millipore, Darmstadt, Germany) absorbance assay by subtracting background readout at $650 \mathrm{~nm}$ from response readout at $570 \mathrm{~nm}$ wavelength. IC50 concentrations were determined after $72 \mathrm{~h}$ of drug treatment between $0.01-100 \mu \mathrm{M}$ in two-fold dilution series. Analysis was performed using CalcuSyn (v2.0, Biosoft, Cambridge, UK).

\section{Melanin quantification}

Melanin pigment production of cultured cells was determined by colorimetric measurements normalized for total protein levels in arbitrary units [28, 29]. Melanoma cells were harvested by centrifugation at $3000 \mathrm{rpm}$ (3830 g, Z326K, Labnet International, Edison, NJ) and dissolved in either $1.0 \mathrm{~N} \mathrm{NaOH}$ for melanin assay or lysis 250 for protein assay. The cell lysates were sonicated, incubated at room temperature for $24 \mathrm{~h}$, and cleared by centrifugation at $13,000 \mathrm{rpm}$ for $10 \mathrm{~min}$ (17,000 g, Z326K, Labnet International, Edison, NJ). The absorption of the supernatant was measured at $475 \mathrm{~nm}$ in a spectrophotometer (Smartspec3000, Bio-Rad, Carlsbad, CA). Cells were lysed in mild denaturing conditions in lysis 250 buffer ( $25 \mathrm{mM}$ Tris, [pH 7.5], $5 \mathrm{mM}$ EDTA, $0.1 \% \mathrm{NP}-40,250 \mathrm{mM} \mathrm{NaCl}$ ) containing proteinase inhibitors $(10 \mu \mathrm{g} / \mathrm{ml}$ aprotinin, $10 \mu \mathrm{g} / \mathrm{ml}$ leupeptin, $10 \mu \mathrm{g} / \mathrm{ml}$ pepstatin, $5 \mu \mathrm{g} / \mathrm{ml}$ antipain, $1 \mathrm{mM}$ phenylmethylsulfonyl fluoride). The total protein amount in the lysates was quantified using a colorimetric Bradford assay (5000001, Bio-Rad, Richmond, CA) at $595 \mathrm{~nm}$ and an incubation time of $30 \mathrm{~min}$ [30].

\section{Results}

\section{Generation of BRAFi-resistant melanoma cell lines}

The parental melanoma cell lines SK-MEL-28 and A375 were exposed to incrementally increasing concentrations of the mutant-BRAF inhibitor vemurafenib (Fig. 1a). At the initial inhibitor concentration matching the IC50 of vemurafenib in the naive parental melanoma cells [11, 31] cell proliferation decreased. Surviving cells were propagated and subjected to an exponential series of increasing vemurafenib concentrations until BRAFi-R sublines were obtained tolerating at least $5 \mu \mathrm{M}$ vemurafenib in the culture media with similar cell proliferation rates as the parental cell lines of 0.67 doublings per day.

Some BRAFi-R cell lines showed structures typically observed in differentiated melanocytes (Fig. 1b-c). In the presence of $5 \mu \mathrm{M}$ vemurafenib, however, the parental cells were not able to grow but the resistant cells proliferated comparable to naïve cell lines (Fig. 1d-e). For the SK-MEL-28 cell line, two resistant sublines were established. The resistant sublines displayed IC50 values of $11.5 \pm 0.9 \mu \mathrm{M}$ and $13.3 \pm 1.2 \mu \mathrm{M}$ for SK-MEL-28-BRAFiR1 and SK-MEL-28-BRAFi-R2 respectively, which is approximately $10-20$ fold of the IC50 in a low micromolar range for the parental cells with $0.74 \pm 0.05 \mu \mathrm{M}$. For the A375 cell line, the IC50 of the A375-BRAFi-R cell line was observed at $17.7 \pm 1.5 \mu \mathrm{M}, 22.7$ fold of IC50 for the parental A375 cells with $0.78 \pm 0.22 \mu \mathrm{M}$ (Fig. 1f). 

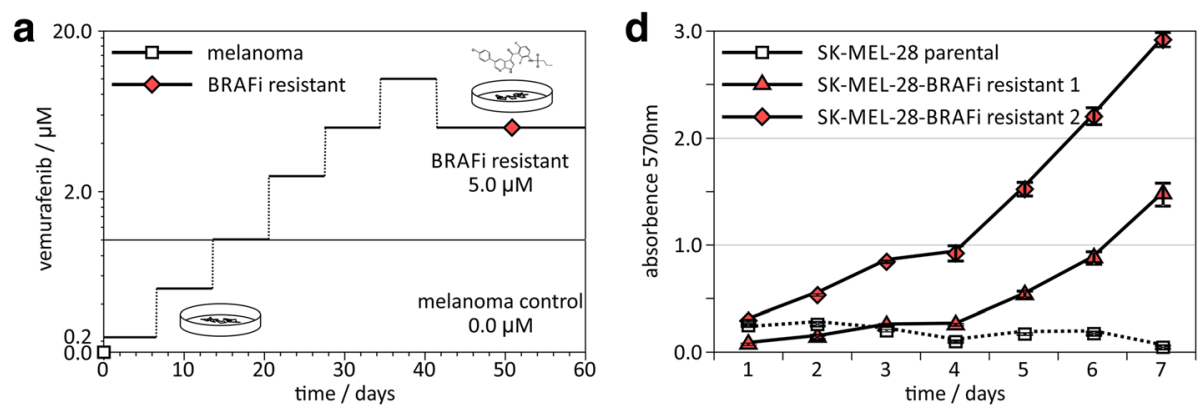

b

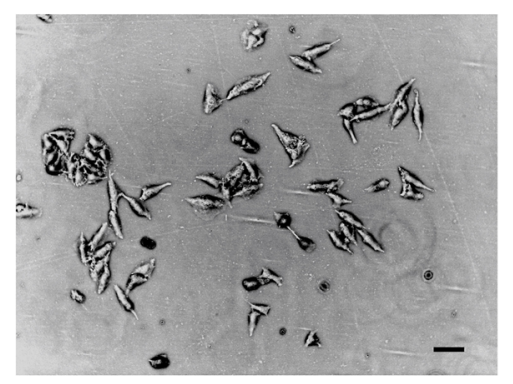

C

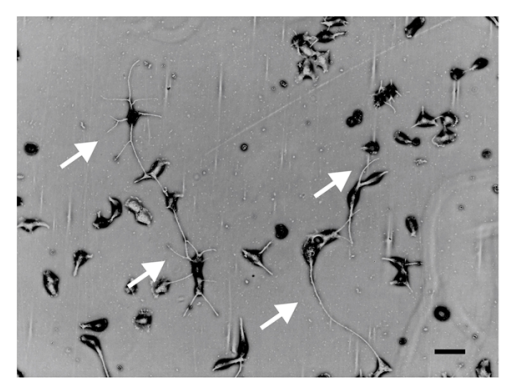

e
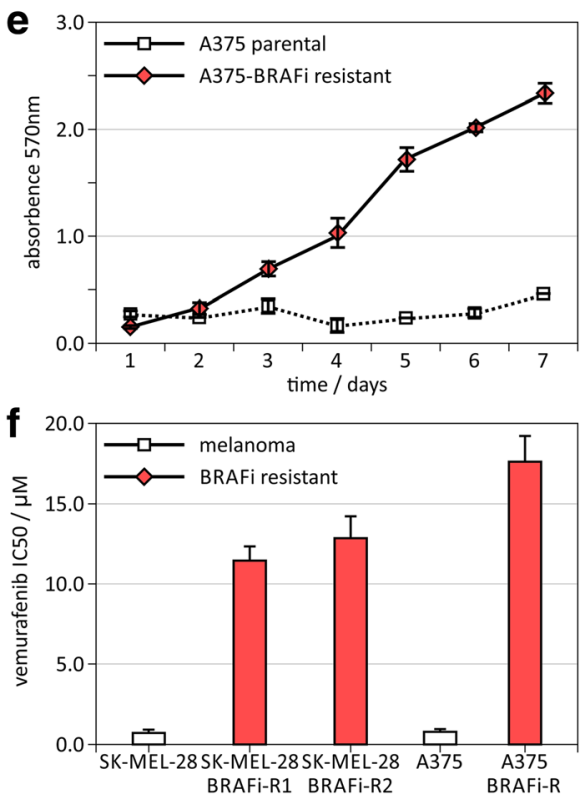

Fig. 1 Establishing mitogen activated protein kinase inhibitor-resistant melanoma models. a A mitogen activated protein kinase BRAF inhibitorresistant (BRAFi-R) model was established using SK-MEL-28 and A375 malignant melanoma cell lines. Schedule of administered concentrations of mitogen activated kinase inhibitor, vemurafenib. b Phase contrast images of control SK-MEL-28 parental melanoma cell lines and (c) BRAF inhibitor-resistant SK-MEL-28-BRAFi-R melanoma cell line 1. Black bar indicates $1.0 \mu \mathrm{m}$. White arrows in image of resistant cell lines point to cellular structures typical for differentiated melanocytes. $\mathbf{d}$ and $\mathbf{e}$ Cell viability assay on melanoma cell lines at $10 \mu \mathrm{M}$ vemurafenib. Absorption in XTT assay is measured at $570 \mathrm{~nm}$. White squares indicate control melanoma cell lines, red triangles and diamonds show melanoma BRAFi-R model. f IC50 concentrations of vemurafenib of control and drug-resistant cancer cell lines

Transcriptomic profiling identifies non-genomic rewiring of treatment-resistant cancer cells

We conducted transcriptomic gene expression profiling of BRAFi treatment-resistant SK-MEL-28-BRAFi-R1 and SK-MEL-28-BRAFi-R2 cell lines by RNA-Seq and looked for differential expression versus the parental SK-MEL28 cell line. In total, 980 unique transcripts showed significant differential expression in RNA-Seq experiments with $p$ values below 0.05 , absolute log-fold change (LOG(FC)) greater or equal 1.0 (Fig. 2a-b). The differentially expressed genes included 505 upregulated transcripts and 475 downregulated transcripts (Additional file 1: Table S2-3). We subjected the identified directional sets to pathway enrichment analysis (Additional file 1: Table S4). Distinct clusters stood out and showed significant enrichment with $p$ values below 0.05 and $q$ values below 0.10 (Fig. 2c). Melanogenesis and pathways in cancer, inflammation, nuclear factor kappa-lightchain-enhancer of activated $\mathrm{B}$ cells $\left(\mathrm{NF}_{\mathrm{B}} \mathrm{B}\right)$ and signal transducer and activator of transcription (STAT) signaling, metabolic pathways including alanine, tyrosine, valine, leucine, inositol, one-carbon metabolism, celladhesion molecules, neurotrophin signaling were overrepresented in the upregulated dataset. MAPK signaling and epithelial-mesenchymal transition (EMT) were differentially expressed and characterized by both strong up- and downregulation. Extra-cellular matrix (ECM) receptors, cell cycle, and hypoxia signaling were enriched in the downregulated dataset. Of the 980 differential expressed genes, we validated expression changes of 150 genes by RT-qPCR (Fig. 2d, Additional file 1: Table S3). Of these, a majority, $64.0 \%$ (96 of 150), responded 


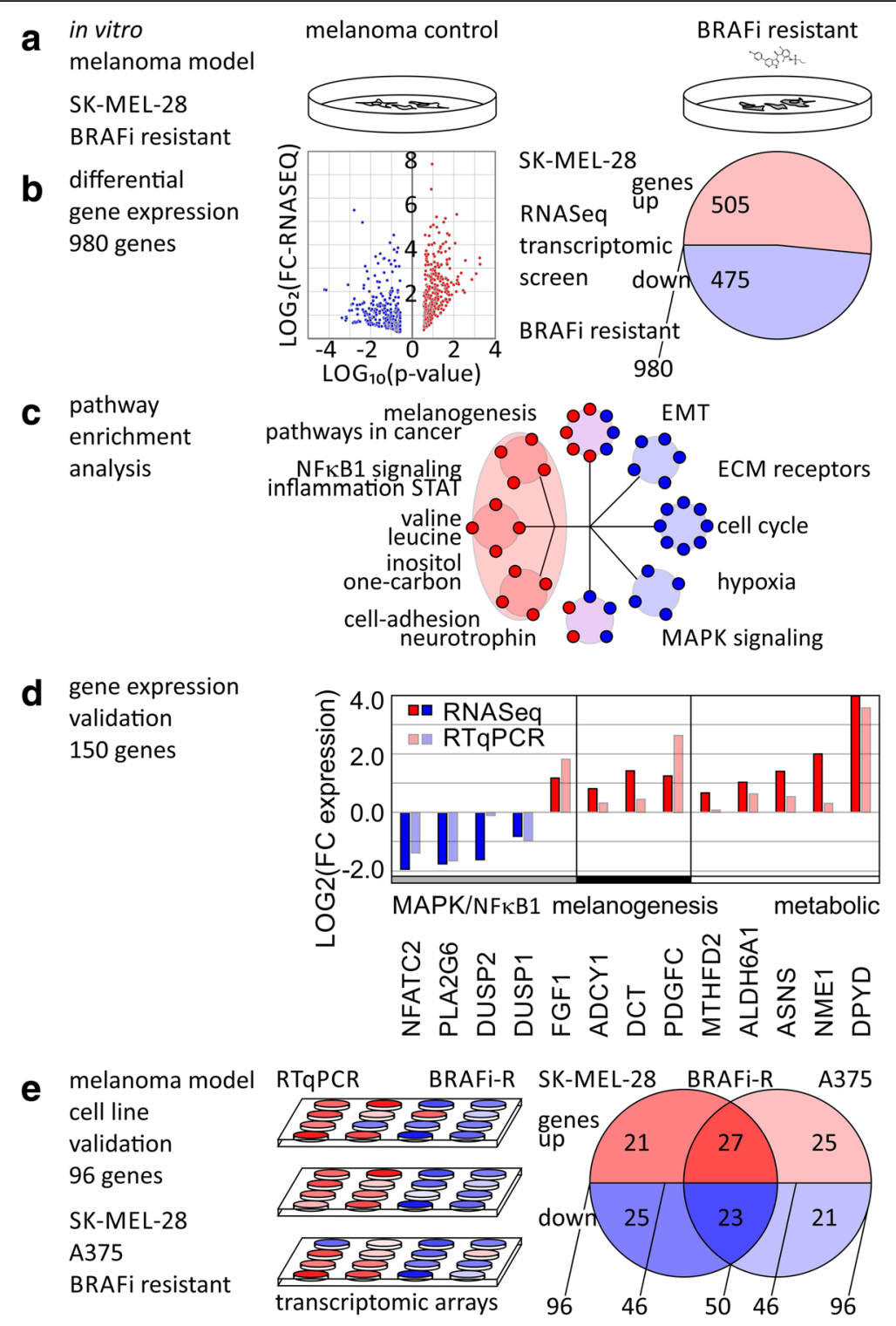

Fig. 2 Transcriptomic profiling of BRAF inhibitor resistance in cellular models of malignant melanoma. a Establishing cellular models of mitogen activated protein kinase inhibitor resistance using SK-MEL-28 malignant melanoma cell line and the BRAF inhibitor, vemurafenib. b Transcriptomics RNA-Seq analysis identifies 980 differentially expressed genes between BRAF inhibitor-resistant (BRAFi-R) cellular model vs control. c Enrichment analysis of up- and downregulated gene sets shows shift in metabolic and signaling pathways. $\mathbf{d}$ Validation by transcriptomic profiling of identified genes by RT-qPCR. e Comparison and validation of resistance model using melanoma cell lines SK-MEL-28 and A375 by transcriptomics RT-qPCR arrays

significantly (with $p$ values below 0.05 ) in the same direction as RNA-Seq data for treatment-resistant melanoma. When both treatment resistance models of SKMEL-28 and A375 were taken into consideration, about half of the tested genes, 50 of 96, showed consistent regulation (Fig. 2e, Additional file 1: Table S3). Genes in MAPK signaling included nuclear factor of activated T-cells 2 (NFATC2, Gene ID: 4773), phospholipase A2 group VI (PLA2G6, Gene ID: 8398), dual specificity phosphatase 1 (DUSP1, Gene ID: 1843), and dual specificity phosphatase 2 (DUSP2, Gene ID: 1844), which were downregulated in the BRAFi-R cells compared to control. Genes contributing to melanogenesis adenylate cyclase 1 (ADCY1, Gene ID: 107), dopachrome tautomerase (DCT, TYRP2, Gene ID: 1638), and platelet derived growth factor C (PDGFC, Gene ID: 56034) were upregulated. Lastly, metabolic regulators such as methylenetetrahydrofolate dehydrogenase 2 (MTHFD2, Gene ID: 10797) for folate metabolism, asparagine synthetase (ASNS, Gene ID: 440) 
for amino acid metabolism, and NME/NM23 nucleoside diphosphate kinase 1 (NME1, Gene ID: 4830) and dihydropyrimidine dehydrogenase (DPYD, Gene ID: 1806) for pyrimidine metabolism were significantly upregulated (Fig. 2d). Taken together, the adaptive transcriptomic changes were validated in two distinct melanoma models, SK-MEL-28 and A375, both cell lines with metastatic potential showed differential expression of MAPK signaling while activating alternative mitogenic signaling interactions and metabolic processes.

\section{Upstream regulator analysis suggests control by transcription factor families}

Next, the gene list was subjected to hierarchical transcription factor motif analysis to identify master regulators [32]. We asked whether any of the enriched transcription factor motif families were represented in the differential gene expression data. In detail, we looked for transcription factors as well as their target genes whose promoters show respective transcription factor binding sites among the same list of regulated genes (Fig. 3a). It is expected that differentially expressed transcription factors show motif enrichment in promoter sites of significantly deregulated target genes. Further, identified target genes with enriched transcription factor motifs will have major contributions to significantly deregulated pathways under treatment resistance (Fig. 3b). A network illustration of transcriptional master regulators, target genes, and dysregulated effector network upon treatment resistance demonstrates transcriptional synergy (Fig. 3c). Upregulated transcription factor families included Rel homology region (RHR) NFkB-related factors, forkhead box (FOX), Zinc finger E-box-binding homeobox domain factors (ZEB), nuclear steroid hormone receptor subfamily 3 (NR3C, androgen receptor and progesterone receptor), hypoxia-inducible and endothelial PAS domain-containing factors (HIF, EPAS), and the cell cycle transcription factor family (E2F) (Fig. 3b). Downstream enriched target genes comprised members of interleukin (IL), chemokine receptor (CXCL), matrix metallo proteinase (MMP) families, transcription factors forkhead box O1 (FOXO1, Gene ID: 2308), endothelial PAS domain protein 1 (EPAS1, HIF2A, Gene ID: 2034) and melanogenesis associated metabolic genes, tyrosinase (TYR, OCA1, Gene ID: 7299), DCT, and melanosomal transmembrane protein (OCA2, oculocutaneous albinism II, Gene ID: 4948). Downregulated transcription factors included forkhead box F2 (FOXF2, Gene ID: 2295), which has DUSP2 or transforming growth factor beta 3 (TGFB3, Gene ID: 7043) as target genes. Upstream regulator analysis suggested gene expression changes of nuclear factor kappa B subunit 1 (NFKB1, Gene ID: 4790, V\$NFKB_Q6, motif M11921) in complex with REL proto-oncogene (REL Gene ID: 5966, V\$CREL_01, motif M10143), EMT modulator zinc finger E-box binding homeobox 1 (ZEB1, Gene ID: 6935, V\$AREB6_01, M11244), forkhead box (V\$FOXO1_01, motif M11512), and hypoxia inducible factor family transcription factors (V\$HIF1_Q3, motif M14011) as master regulators of transcriptional effector networks upon BRAFi treatment resistance.

\section{Validation of pathway rewiring in drug resistance in multiple cell lines by transcriptomics arrays}

Transcriptome analysis of reversible drug resistance identified distinct pathways that allowed for circumvention of BRAF blockage (Fig. 4a). Cell-to-cell variability in combination with drug exposure selects for distinct subpopulations of MAPKi-resistant (MAPKi-R) cell lines. In a hierarchical fashion, transcriptional master regulators promote a distinct set of target genes resulting in circumvention of MAPK inhibition. Receptor activation by fibroblast growth factor 1 (FGF1, Gene ID: 2246) or PDGFC can lead to activated receptor tyrosine signaling parallel to canonical MAPK signaling [16] (Fig. 4b). In addition, downregulation of tumor suppressors reengages mitogenic signaling. The dual specific phosphatases, DUSP1 and DUSP2, have the ability to switch MAPK signaling off and rank among the top downregulated hits. Thus, downregulation of dual specific phosphatases facilitates and reinforces alternative MAPK effector activation under BRAF blockage (Fig. 4b). One of the mitogen-activated protein kinase 1 (MAPK1, ERK2, Gene ID: 5594) effector targets, transcription factor EPAS1, showed upregulation and the ability to maintain its transcriptional program. The pro-apoptotic program of TGFB3 was downregulated including SMAD family member 9 (SMAD9, Gene ID: 4093) and DUSP1/2 (Fig. 4c). Adenylate cyclase, G-protein, and phospholipase signaling are alternative cascades observed in cutaneous and uveal melanoma (Fig. 4d). Upregulation of ADCY1, endothelin receptor type B (EDNRB, Gene ID: 1910), phospholipase C beta 4 (PLCB4, Gene ID: 5332), and cAMP responsive element binding protein 3 (CREB3, Gene ID: 10488) promote MITF activity, the master transcription factor for pigmentation genes. Downstream metabolic enzymes, TYR and DCT, are both MITF target genes and contribute to enhanced eumelanin production observed in some therapy-resistant cell lines. The observed pigmentation showed a wide range of from 1.3-fold to up to 16.8 -fold upregulation (Fig. 4d). While both cell lines showed dysregulation of melanogenesis, the regulators and effectors involved were different. SK-MEL-28BRAFi-R2 has ASIP prominently expressed (TYR (2.1), DCT (2.8), tyrosinase related protein 1 (TYRP1, OCA3, Gene ID: 7306) (0.5), MITF (0.7), agouti signaling protein (ASIP, Gene ID: 434) (18.9)), while A375-BRAFi-R showed strongest regulation of TYRP1 and MITF (TYR (0.34), DCT (0.24), TYRP1 (41.8), MITF (2.94), ASIP (0.41)). 

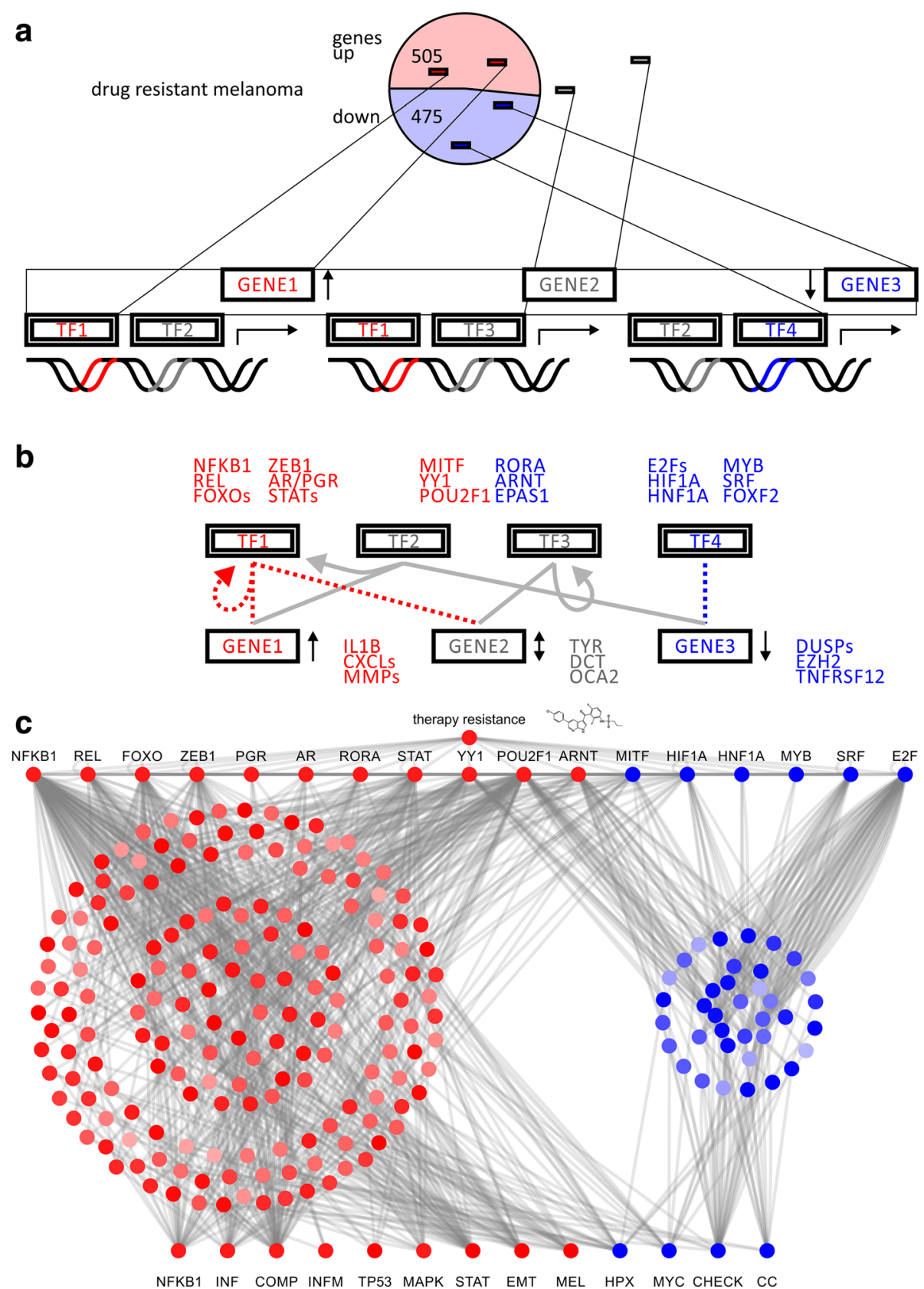

Fig. 3 Transcription factor motif analysis of mitogen activated protein kinase inhibitor resistance in cellular models of malignant melanoma. a Schematic representation of differentially expressed genes in drug resistance model and transcription factor motifs associated with regulated target genes. Upregulated and downregulated factors are depicted in red and blue, respectively. b Hierarchical transcription factor network with master regulators on top and downstream targets at bottom. Sets of transcription factor target genes are identified in enrichment analysis based on sequence motifs. c Hierarchical network model illustrates how therapy resistance in cancer selects for specific transcriptional master regulators to rewire target genes in effector pathways in a concerted fashion

In summary, upregulation of growth factors or receptors triggers signaling pathways circumventing BRAF blockage. Changes in amino acid and one-carbon metabolism support cellular proliferation despite inhibitor treatment. In addition, alternative MAPK signaling coincides with differential response of melanogenesis and pigmentation pathways, which partially overlap with MAPK effectors. In particular, NFKB1, REL, ZEB1, FOXO1, and EPAS1 may serve as master regulators to enact broad transcriptional changes implemented in altered cascades of MAPK, TGFB, ADCY, and MITF signaling. 


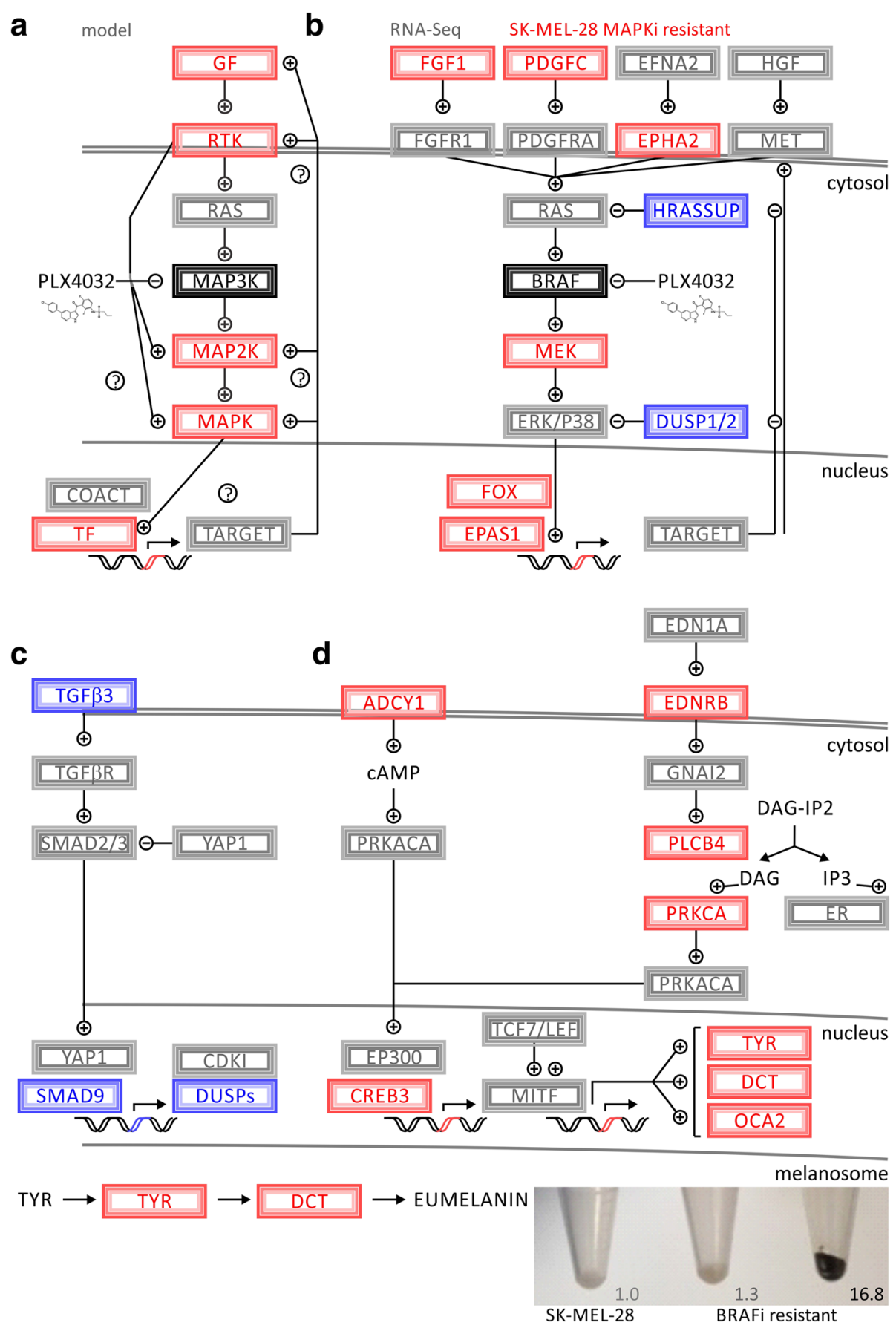

Fig. 4 Pathway analysis of BRAF kinase inhibitor resistance shows alternative activation of MAPK targets and pigmentation. a Schematic representation of regulatory network involving drug inhibition and non-genomic selection for differential expression of driver genes that can circumvent suppressed signaling. $\mathbf{b}$ Deregulation of MAPK signaling with RNA-Seq data is mapped in red and blue for differential upregulation and downregulation, respectively. c Modulation of TGFB signaling leads to downregulation of dual specific phosphatases, which are required to switch MAPK signaling off. d Interconnectedness between G-protein signaling and melanogenesis. Alternative activation of melanoma pathways leads to increased eumelanin synthesis and mitogenic survival. Photograph of cell pellets of melanoma cell models and detected melanin. Left shows SK-MEL-28 melanoma cell line, middle and right shows two different SK-MEL-28-BRAFi-resistant melanoma cell lines with elevated melanin production

\section{Discussion}

Activation of the MAPK pathway is the central and most common oncogenic event in the pathogenesis of malignant melanoma [3, 33]. About $50 \%$ of all melanoma patients have activating somatic mutations in the activator loop involving L597, T599, V600, and K601 
switching proto-oncogene BRAF into a constitutively active protein kinase and cancer driver. Such activation is supported by somatic copy number amplifications of chromosome 7 [34], often coinciding with somatic V600E/G/K/M/R mutations. Another $20-30 \%$ of the patients show nongenomic activation of BRAF by transcriptional upregulation or post-translational modification induced by somatic mutations of upstream signaling molecules like KIT protooncogene receptor tyrosine kinase (KIT, Gene ID: 3815), proto-oncogene neuroblastoma RAS viral oncogene homo$\log$ (NRAS, Gene ID: 4893), or loss-of-function neurofibromin 1 (NF1, Gene ID: 4763). Constitutively activated BRAF phosphorylates MAPK1 and downstream kinases resulting in mitogenic signaling, proliferation, and cell growth. Integrated into this cellular program is negative feedback resulting in reduction of NRAS expression [35, 36].

\section{Genomic and non-genomic mechanisms of therapy resistance}

Genomic sequencing has facilitated the understanding of acquired resistance mechanisms to MAPKis [14-16, 37-40]. Detected genetic aberrations included mutations in NRAS, MAPK1/2, phosphatidylinositol-4,5-bisphosphate 3-kinase catalytic subunit alpha (PIK3CA, Gene ID: 5290), and phosphatase and tensin homolog (PTEN, Gene ID: 5728). Somatic melanoma mutations provide examples of how single, well-defined genomic events can confer resistance against vemurafenib treatment. In contrast, transcriptomic as well as epigenomic regulation can provide insight into resistance states that may involve larger networks. Eventually, resistance-conferring genomic, epigenomic, and transcriptomic alterations result in sustained mitogenic effector signaling and persist to promote proliferation.

\section{Network rewiring of therapy-resistant melanoma}

The transcriptomic profiles revealed a network of genes involved in adenylate cyclase signaling conferring resistance and contributing to melanogenesis. ADCY1 and CREB3 are prominent members of the melanogenesis pathway exhibiting mitogenic control and MITF activation. Similarly, a gain-of-function screen confirmed a cyclic-AMP-dependent melanocytic signaling network including G-protein-coupled receptors, adenylate cyclase, protein kinase cAMP-activated catalytic subunit alpha (PRKACA, Gene ID: 5566), and cAMP responsive element binding protein 1 (CREB1, Gene ID: 1385) [41]. The MAPK pathway negatively regulates MITF protein level as well as activity [29], which in turn regulates a series of cell cycle regulating genes. In particular, P16INK4A and P21CIP1, gene products of cyclin dependent kinase inhibitor 2A (CDKN2A, Gene ID: 1029) and cyclin dependent kinase inhibitor 1A (CDKN1A, Gene ID: 1026), respectively, differentiation genes TYR, DCT, TYRP1 as well as survival genes B-cell lymphoma 2 apoptosis regulator
(BCL2, Gene ID: 596) and BCL2 family apoptosis regulator (MCL1, Gene ID: 4170) are effector genes under the control of MITF. Indeed, inhibition of MITF increases sensitivity to chemotherapy drugs [42]. In contrast, upregulation of MITF in therapy-resistance may present itself as a survival mechanism, which coincides with upregulation of melanin, hence it may serve as prognostic biomarker for drug adaptation.

Dual specific phosphatases (DUSPs) act downstream of BRAF on phosphorylated MAPK members to provide attenuation of signal. Loss of DUSP activity results in constitutive activation of the pathway. Prominent members of this family DUSP1 and DUSP2 are consistently downregulated at the transcriptional level. In prior clinical studies, somatic mutation of DUSP4 in MAPKi-R has been reported [39]. Although in that case a genomic mechanism of resistance was utilized, the outcome of reduced DUSP activity by genomic or transcriptomic changes is equivalent and leads to persistent triggering of MAPK effectors.

\section{Metabolic support of therapy resistance}

Metabolic genes support the rewiring of acquired resistance and have been shown to play an intricate role in the malignancy of skin cutaneous tissues. Glutamine and glucose metabolism showed sensitivity to combinations of MAPKi and metabolic inhibitors in preclinical studies [43]. The transciptomic profiles identified key enzymes in related, branching glycolytic pathways of serine, folate and pyrimidine metabolism. A cancer systems biology analysis of skin cutaneous melanoma brought forward a new master regulator and diagnostic target in cancer metabolism. Somatic mutations of DPYD have the ability to reconfigure and activate pyrimidine metabolism promoting rapid cellular proliferation and metastatic progression [44].

\section{Concertation of transcriptional regulators}

The forkhead box family of transcription factors is an important downstream target of the MAPK pathway and is currently being considered as a new therapeutic target in cancer, including melanoma therapy [45]. In epithelial cells, these transcriptional factors are directly involved in the expression of cyclin dependent kinase inhibitors and CDKN2A gene under the control of TGF $\beta[46,47]$. Both downregulation of anti-apoptotic targets as well as activation of proliferative metabolism have been observed as mechanisms contributing to MAPKi-R. Downregulation of FOXF2 has been shown to promote cancer progression, EMT, and metastatic invasion [48]. In contrast, a different member of the FOX family, the stem cell transcription factor forkhead box D3 (FOXD3) has been identified as an adaptive mediator of the response to MAPK pathway inhibition selectively in mutant BRAF melanomas $[49,50]$.

We have discovered non-genomic rewiring of pathways in chemotherapy resistance by RNA-Seq data and 
validated gene targets in two cell lines by transcriptomics arrays. Perturbation of these resistance pathways by drug molecules, RNA interference, or genomic editing will corroborate the translational impact of identified gene targets. The established cell culture models of treatment resistance provide a broadly applicable platform to utilize high-throughput screening tools in the search for effective combinations of targeted therapies in cancer.

\section{Conclusion}

The MAPK pathway undergoes major rewiring at the transcriptional level while acquiring inhibitor resistance. The outcome of such transcriptional plasticity is dysregulation at the level of different upstream master regulators, while maintaining similar effector genes. Combination therapies including targeted approaches and immune checkpoint inhibition are promising and rapidly improving. For these therapies to show durable, progression-free success in the clinical setting, adaptation mechanisms of treatment resistance need to be understood. Cellular model systems in combination with transcriptome-wide analyses provide insight into how non-genomic drug adaptation is accomplished. Ongoing efforts are focused on utilizing the established preclinical models to overcome drug adaptation as well as precision medicine profiling of cancer patients. Over time, a better understanding of mechanisms involved in drug adaptation is likely to improve the effectiveness of melanoma therapy by delaying or controlling acquired resistance.

\section{Additional file}

Additional file 1: Table S1-S4 are compiled as supplementary information. Table S1: Oligonucleotides for RT-qPCR arrays. Table S2: Differentially expressed gene set based on RNA-Seq data. Table S3: Validated transcripts. Table S4: enrichment based on directional transcriptomic data. (XLSX $94 \mathrm{~kb}$ )

\section{Abbreviations \\ BRAF: B-Raf proto-oncogene, serine/threonine kinase; BRAFi: BRAF inhibitor; BRAFi-R: BRAFi-resistant; CDNA: complementary DNA; CT: threshold cycle; DMSO: dimethyl sulfoxide; DUSPs: dual specific phosphatase; ECM: extra- cellular matrix; EMT: epithelial-mesenchymal transition; FOX: forkhead box; HIF: hypoxia-inducible factor; IC50: half maximal inhibitory concentration; LOG(FC): log-fold change; MAPK: mitogen activated protein kinase; MAPKi: MAPK inhibitor; MAPKi-R: MAPKi-resistant; MEKi: MEK inhibitor; XTT: 2,3-bis(2-methoxy-4-nitro-5-sulfophenyl)-2H-tetrazolium-5-carboxanilide; RNA-Seq: next generation mRNA transcriptome sequencing; RPKM: RNA-Seq single-end reads in reads per kilobase million; RT-qPCR: real-time quantitative polymerase chain reaction; STAT: signal transducer and activator of transcription}

\section{Acknowledgements}

We would like to thank Angela Garcia, Charles Fagundes, Garja Suner, Sandeep Sanghera, Taran Kaur, Kirandeep Kaur, Keedrian Olmstead, Stephen Wilson, and Rohit Gupta for help with maintaining the cellular models of drug-resistant cancer cells.

\section{Availability of preprint publication}

The manuscript was made publically available to the scientific community [51] on the preprint server bioRxiv under doi: https://doi.org/10.1101/231142 following submission March 1, 2017.

\section{Funding}

FVF is grateful for the support of grant CA154887 from the National Institutes of Health, National Cancer Institute. The research of the University of California Merced Systems Biology and Cancer Metabolism Laboratory is generously supported by University of California, Cancer Research

Coordinating Committee CRN-17-427258, National Science Foundation, University of California Senate Graduate Research Council, and Health Science Research Institute program grants. FLS is supported by grant CA160756 from the National Institutes of Health, National Cancer Institute. FLM and FLS are in part supported by the Waltmar and Oxnard Foundations and Aldrich Chair Endowment.

\section{Authors' contributions}

Conception and design: FLS, FVF Establishing of cellular models, data acquisition, and analysis of data: HZ, DT, ZW, AF, PP, JL, SS, AS, AB, SYT, FLM, FLS,

FVF. Preparation of figures, data analysis, interpretation, writing, review, and revision of the manuscript: FVF. Study supervision: FLS, FVF. All authors read and approved the final manuscript.

\section{Ethics approval and consent to participate}

All experimental protocols were approved by the Institutional Review Boards at the University of California Merced and Irvine. The study was carried out as part of IRB UCM13-0025 of the University of California Merced and as part of dbGap ID 5094 for study accession phs000178.v9.p8 on somatic mutations in cancer and conducted in accordance with the Helsinki Declaration of 1975.

\section{Competing interests}

There is no competing financial interest. FLM is co-Founder and Medical director of Cancer Prevention Pharmaceuticals with no direct implications on the conducted study on melanoma resistance.

\section{Publisher's Note}

Springer Nature remains neutral with regard to jurisdictional claims in published maps and institutional affiliations.

\section{Author details}

'Systems Biology and Cancer Metabolism, Program for Quantitative Systems Biology, University of California Merced, 2500 North Lake Road, Merced, CA 95343, USA. ${ }^{2}$ Department of Medicine, School of Medicine, Chao Family Comprehensive Cancer Center, University of California Irvine, Irvine, CA 92697, USA. ${ }^{3}$ The State Key Laboratory of Medical Genetics and School of Life Sciences, Department of Molecular Biology, Central South University, Changsha 410078, China. ${ }^{4}$ Department of Epidemiology, School of Medicine, University of California, Irvine, CA 92697, USA.

Received: 29 October 2017 Accepted: 21 February 2018 Published online: 04 April 2018

\section{References}

1. Davies H, Bignell GR, Cox C, Stephens P, Edkins S, Clegg S, et al. Mutations of the BRAF gene in human cancer. Nature. 2002;417(6892):949-54. https:// doi.org/10.1038/nature00766.

2. Pollock PM, Harper UL, Hansen KS, Yudt LM, Stark M, Robbins CM, et al. High frequency of BRAF mutations in nevi. Nat Genet. 2003;33(1):19-20. https://doi.org/10.1038/ng1054.

3. Guan J, Gupta R, Filipp FV. Cancer systems biology of TCGA SKCM: efficient detection of genomic drivers in melanoma. Sci Rep. 2015;5:7857. https://doi. org/10.1038/srep07857.

4. Tsai J, Lee JT, Wang W, Zhang J, Cho H, Mamo S, et al. Discovery of a selective inhibitor of oncogenic B-Raf kinase with potent antimelanoma activity. Proc Natl Acad Sci U S A. 2008;105(8):3041-6. https://doi.org/10. 1073/pnas.0711741105.

5. Chapman PB, Hauschild A, Robert C, Haanen JB, Ascierto P, Larkin J, et al. Improved survival with vemurafenib in melanoma with BRAF V600E mutation. N Engl J Med. 2011;364(26):2507-16. https://doi.org/10.1056/ NEJMoa1103782. 
6. Flaherty KT, Robert C, Hersey P, Nathan P, Garbe C, Milhem M, et al. Improved survival with MEK inhibition in BRAF-mutated melanoma. N Engl J Med. 2012;367(2):107-14. https://doi.org/10.1056/NEJMoa1203421.

7. Flaherty KT, Infante JR, Daud A, Gonzalez R, Kefford RF, Sosman J, et al. Combined BRAF and MEK inhibition in melanoma with BRAF V600 mutations. N Engl J Med. 2012;367(18):1694-703. https://doi.org/10.1056/ NEJMoa1210093.

8. Robert C, Karaszewska B, Schachter J, Rutkowski P, Mackiewicz A, Stroiakovski D, et al. Improved overall survival in melanoma with combined dabrafenib and trametinib. N Engl J Med. 2015;372(1):30-9. https://doi.org/ 10.1056/NEJMoa1412690

9. Long GV, Stroyakovskiy D, Gogas H, Levchenko E, de Braud F, Larkin J, et al. Combined BRAF and MEK inhibition versus BRAF inhibition alone in melanoma. N Engl J Med. 2014;371(20):1877-88. https://doi.org/10.1056/ NEJMoa1406037.

10. Cheng Y, Zhang G, Li G. Targeting MAPK pathway in melanoma therapy. Cancer Metastasis Rev. 2013;32(3-4):567-84. https://doi.org/10.1007/s10555013-9433-9

11. Nazarian R, Shi H, Wang Q, Kong X, Koya RC, Lee H, et al. Melanomas acquire resistance to B-RAF(V600E) inhibition by RTK or N-RAS upregulation. Nature. 2010;468(7326):973-7. https://doi.org/10.1038/nature09626.

12. Wagle N, Emery C, Berger MF, Davis MJ, Sawyer A, Pochanard P, et al. Dissecting therapeutic resistance to RAF inhibition in melanoma by tumor genomic profiling. J Clin Oncol. 2011;29(22):3085-96. https://doi.org/10. 1200/JCO.2010.33.2312

13. Poulikakos PI, Persaud Y, Janakiraman M, Kong X, Ng C, Moriceau G, et al. RAF inhibitor resistance is mediated by dimerization of aberrantly spliced BRAF(V600E). Nature. 2011;480(7377):387-90. https://doi.org/10.1038/ nature10662.

14. Van Allen EM, Wagle N, Sucker A, Treacy DJ, Johannessen CM, Goetz EM, et al. The genetic landscape of clinical resistance to RAF inhibition in metastatic melanoma. Cancer Discov. 2014;4(1):94-109. https://doi.org/10. 1158/2159-8290.CD-13-0617.

15. Moriceau G, Hugo W, Hong A, Shi H, Kong X, Yu CC, et al. Tunablecombinatorial mechanisms of acquired resistance limit the efficacy of BRAF/ MEK cotargeting but result in melanoma drug addiction. Cancer Cell. 2015; 27(2):240-56. https://doi.org/10.1016/j.ccell.2014.11.018.

16. Hugo W, Shi H, Sun L, Piva M, Song C, Kong X, et al. Non-genomic and immune evolution of melanoma acquiring MAPKi resistance. Cell. 2015; 162(6):1271-85. https://doi.org/10.1016/j.cell.2015.07.061.

17. Emmons MF, Faiao-Flores F, Smalley KS. The role of phenotypic plasticity in the escape of cancer cells from targeted therapy. Biochem Pharmacol. 2016 122:1-9. https://doi.org/10.1016/jbcp201606.014.

18. Lito P, Rosen N, Solit DB. Tumor adaptation and resistance to RAF inhibitors. Nat Med. 2013;19(11):1401-9. https://doi.org/10.1038/nm.3392.

19. Carey TE, Takahashi T, Resnick LA, Oettgen HF, Old LJ. Cell surface antigens of human malignant melanoma: mixed hemadsorption assays for humoral immunity to cultured autologous melanoma cells. Proc Natl Acad Sci U S A. 1976;73(9):3278-82.

20. Fogh J, Fogh JM, Orfeo T. One hundred and twenty-seven cultured human tumor cell lines producing tumors in nude mice. J Natl Cancer Inst. 1977; 59(1):221-6

21. Giard DJ, Aaronson SA, Todaro GJ, Arnstein P, Kersey JH, Dosik H, et al. In vitro cultivation of human tumors: establishment of cell lines derived from a series of solid tumors. J Natl Cancer Inst. 1973;51(5):1417-23.

22. Xing F, Persaud Y, Pratilas CA, Taylor BS, Janakiraman M, She QB, et al. Concurrent loss of the PTEN and RB1 tumor suppressors attenuates RAF dependence in melanomas harboring (V600E)BRAF. Oncogene. 2012;31(4): 446-57. https://doi.org/10.1038/onc.2011.250.

23. Langmead B, Salzberg SL. Fast gapped-read alignment with bowtie 2. Nat Methods. 2012;9(4):357-9. https://doi.org/10.1038/nmeth.1923.

24. Trapnell C, Roberts A, Goff L, Pertea G, Kim D, Kelley DR, et al. Differential gene and transcript expression analysis of RNA-seq experiments with TopHat and cufflinks. Nat Protoc. 2012;7(3):562-78. https://doi.org/10.1038/ nprot.2012.016.

25. Robinson MD, McCarthy DJ, Smyth GK. edgeR: a Bioconductor package for differential expression analysis of digital gene expression data. Bioinformatics. 2010;26(1):139-40. https://doi.org/10.1093/bioinformatics/ btp616.

26. Mootha VK, Lindgren CM, Eriksson KF, Subramanian A, Sihag S, Lehar J, et al. PGC-1alpha-responsive genes involved in oxidative phosphorylation are coordinately downregulated in human diabetes. Nat Genet. 2003;34(3):26773. https://doi.org/10.1038/ng1180

27. Subramanian A, Tamayo P, Mootha VK, Mukherjee S, Ebert BL, Gillette MA, et al. Gene set enrichment analysis: a knowledge-based approach for interpreting genome-wide expression profiles. Proc Natl Acad Sci U S A. 2005;102(43):15545-50. https://doi.org/10.1073/pnas.0506580102.

28. Friedmann PS, Gilchrest BA. Ultraviolet radiation directly induces pigment production by cultured human melanocytes. J Cell Physiol. 1987;133(1):8894. https://doi.org/10.1002/jcp.1041330111.

29. Liu F, Singh A, Yang Z, Garcia A, Kong Y, Meyskens FL, Jr. MiTF links Erk1/2 kinase and p21 CIP1/WAF1 activation after UVC radiation in normal human melanocytes and melanoma cells. Mol Cancer 2010; 9:214. doi:https://doi. org/10.1186/1476-4598-9-214.

30. Bradford MM. A rapid and sensitive method for the quantitation of microgram quantities of protein utilizing the principle of protein-dye binding. Anal Biochem. 1976;72:248-54. doi: S0003269776699996 [pii]

31. Sondergaard JN, Nazarian R, Wang Q, Guo D, Hsueh T, Mok S, et al. Differential sensitivity of melanoma cell lines with BRAFV600E mutation to the specific Raf inhibitor PLX4032. J Transl Med. 2010;8:39. https://doi.org/10. 1186/1479-5876-8-39.

32. Filipp FV. Epioncogenes in cancer-identification of epigenomic and transcriptomic cooperation-networks by multi-omics integration of ChIP-Seq and RNA-Seq data. Syst Biol. Meth Mol Biol. 2019;1800:101-21.

33. Shain AH, Bastian BC. From melanocytes to melanomas. Nat Rev Cancer. 2016;16(6):345-58. https://doi.org/10.1038/nrc.2016.37.

34. Tiffen J, Wilson S, Gallagher SJ, Hersey P, Filipp FV. Somatic copy number amplification and Hyperactivating somatic mutations of EZH2 correlate with DNA methylation and drive epigenetic silencing of genes involved in tumor suppression and immune responses in melanoma. Neoplasia. 2016;18(2): 121-32. https://doi.org/10.1016/..neo.2016.01.003.

35. Lito P, Pratilas CA, Joseph EW, Tadi M, Halilovic E, Zubrowski M, et al. Relief of profound feedback inhibition of mitogenic signaling by RAF inhibitors attenuates their activity in BRAFV600E melanomas. Cancer Cell. 2012;22(5): 668-82. https://doi.org/10.1016/j.ccr.2012.10.009.

36. Yao Z, Torres NM, Tao A, Gao Y, Luo L, Li Q, et al. BRAF mutants evade ERKdependent feedback by different mechanisms that determine their sensitivity to pharmacologic inhibition. Cancer Cell. 2015;28(3):370-83. https://doi.org/10.1016/j.ccell.2015.08.001.

37. Wagle N, Van Allen EM, Treacy DJ, Frederick DT, Cooper ZA, Taylor-Weiner A, et al. MAP kinase pathway alterations in BRAF-mutant melanoma patients with acquired resistance to combined RAF/MEK inhibition. Cancer Discov. 2014;4(1):61-8. https://doi.org/10.1158/2159-8290.CD-13-0631.

38. Shi H, Hugo W, Kong X, Hong A, Koya RC, Moriceau G, et al. Acquired resistance and clonal evolution in melanoma during BRAF inhibitor therapy. Cancer Discov. 2014;4(1):80-93. https://doi.org/10.1158/2159-8290.CD-130642.

39. Johnson DB, Menzies AM, Zimmer L, Eroglu Z, Ye F, Zhao S, et al. Acquired BRAF inhibitor resistance: a multicenter meta-analysis of the spectrum and frequencies, clinical behaviour, and phenotypic associations of resistance mechanisms. Eur J Cancer. 2015;51(18):2792-9. https://doi.org/10.1016/j.ejca. 2015.08.022.

40. Filipp FV. Precision medicine driven by cancer systems biology. Cancer Metastasis Rev. 2017;36(1):91-108.. https://doi.org/10.1007/s10555-017-9662-4.

41. Johannessen CM, Johnson LA, Piccioni F, Townes A, Frederick DT, Donahue MK, et al. A melanocyte lineage program confers resistance to MAP kinase pathway inhibition. Nature. 2013;504(7478):138-42. https://doi.org/10.1038/ nature12688.

42. Garraway LA, Widlund HR, Rubin MA, Getz G, Berger AJ, Ramaswamy S, et al. Integrative genomic analyses identify MITF as a lineage survival oncogene amplified in malignant melanoma. Nature. 2005;436(7047):117-22. https://doi.org/10.1038/nature03664

43. Hernandez-Davies JE, Tran TQ, Reid MA, Rosales KR, Lowman XH, Pan M, et al. Vemurafenib resistance reprograms melanoma cells towards glutamine dependence. J Transl Med. 2015;13:210. https://doi.org/10.1186/ s12967-015-0581-2.

44. Edwards L, Gupta R, Filipp FV. Hypermutation of DPYD deregulates pyrimidine metabolism and promotes malignant progression. Mol Cancer Res. 2016;14(2):196-206. https://doi.org/10.1158/1541-7786.MCR-15-0403.

45. Yang JY, Hung MC. A new fork for clinical application: targeting forkhead transcription factors in cancer. Clin Cancer Res. 2009;15(3):752-7. https://doi. org/10.1158/1078-0432.CCR-08-0124. 
46. Gomis RR, Alarcon C, He W, Wang Q, Seoane J, Lash A, et al. A FoxO-Smad synexpression group in human keratinocytes. Proc Natl Acad Sci U S A. 2006;103(34):12747-52. https://doi.org/10.1073/pnas.0605333103.

47. Lasfar A, Cohen-Solal KA. Resistance to transforming growth factor betamediated tumor suppression in melanoma: are multiple mechanisms in place? Carcinogenesis. 2010;31(10):1710-7. https://doi.org/10.1093/carcin/ bgq155.

48. Kong PZ, Li GM, Tian Y, Song B, Shi R. Decreased expression of FOXF2 as new predictor of poor prognosis in stage I non-small cell lung cancer. Oncotarget. 2016;7(34):55601-10. https://doi.org/10.18632/oncotarget.10876.

49. Abel EV, Aplin AE. FOXD3 is a mutant B-RAF-regulated inhibitor of G(1)-S progression in melanoma cells. Cancer Res. 2010;70(7):2891-900. https://doi. org/10.1158/0008-5472.CAN-09-3139.

50. Abel EV, Basile KJ, Kugel CH 3rd, Witkiewicz AK, Le K, Amaravadi RK, et al. Melanoma adapts to RAF/MEK inhibitors through FOXD3-mediated upregulation of ERBB3. J Clin Invest. 2013;123(5):2155-68. https://doi.org/10. $1172 / \mathrm{JCl} 65780$.

51. Zecena H, Tveit D, Wang Z, Farhat A, Panchal P, Liu J, et al. Systems biology analysis of mitogen activated protein kinase inhibitor resistance in malignant melanoma bioRxiv; 2017. https://doi.org/10.1101/231142.

Submit your next manuscript to BioMed Central and we will help you at every step:

- We accept pre-submission inquiries

- Our selector tool helps you to find the most relevant journal

- We provide round the clock customer support

- Convenient online submission

- Thorough peer review

- Inclusion in PubMed and all major indexing services

- Maximum visibility for your research

Submit your manuscript at www.biomedcentral.com/submit
Biomed Central 